\title{
Benito... a propósito de Josué. Un estudio fenomenológico del duelo por la muerte de un hijo.
}

Alfonso Miguel García Hernández 1,

1. Profesor titular. Departamento de Enfermería. Universidad de La Laguna. Islas Canarias. España. Grupo de investigación. Universidad de La Laguna "Cuidados al final de la vida (CUFINVIDA)". Tenerife. España.

Recibido: 07 de marzo de 2019

Aceptado: 04 de abril de 2019

\section{Resumen:}

El presente trabajo se engloba en la investigación cualitativa, desde el paradigma hermenéutico-fenomenológico. Realizado desde un análisis narrativo de los textos y las conversaciones compartidas con Benito a lo largo de los últimos años, incorpora y analiza los textos escritos y relatados como testimonios, en términos de experiencias sentidas, manifestadas y vividas, de modo que sitúa a las categorías 
explicativas en un marco más amplio que se haya inserto en la continuidad de vínculos con el hijo fallecido.

El camino del duelo que nos acerca Benito profundiza, por un lado, en las ideas asociadas al sinsentido y el dolor por la ausencia del hijo fallecido, y por otro transita entre el plano de vivir, recordar, trascender y realizar determinadas prácticas socioculturales que le permiten seguir conectado con su hijo Josué, fallecido a consecuencia de un accidente de tráfico. Todo en un escenario en el que las narrativas revelan la naturaleza de los encuentros de conexión y de interacción.

Palabras clave: duelo, muerte de hijo, fenomenología, continuidad de vínculos, Facebook.

\section{Abstract:}

The current work is encompassed in qualitative research, from the hermeneutic-phenomenological paradigm perspective. It is carried out from a narrative analysis of the texts and conversations shared with Benito over the last few years. It incorporates and analyses the texts written and related as testimonies, in terms of experiences felt, manifested and lived, in such a way, that it situates the explanatory categories in a broader framework that forms part of the continuity of links with the deceased son.

Benito moves us closer to the path of grief deepening, on the one hand, into the ideas associated with nonmeaning and pain for the absence of the deceased son and, on the other hand, going through the plane of living, remembering, transcending and carrying out certain socio-cultural practices. They allow him to remain connected to his son Josué, who died as a consequence of a traffic accident. And all of it is developed in a scenario in which the narratives reveal the nature of the encounters of connection and interaction.

Keywords: grief, death of a son, phenomenology, continuing bonds, Facebook. 


\section{Desarrollo}

Conocí a Benito ${ }^{1}$ y a su esposa en enero de 2011, me los presentaron tras el funeral de su hijo y posteriormente tuvimos una veintena de encuentros individuales y grupales en los que compartimos distintas vivencias y experiencias relacionadas con la vida, muerte y duelo por su hijo, el presente artículo agrupa fundamentalmente fragmentos relativos a su diario y a los comentarios compartidos en su Facebook personal.

Benito perdió a su hijo Josué de veinte años de edad a consecuencia de un accidente de tráfico la mañana del día 1 de enero de 2011 cuando salía de la fiesta de fin de año. A dicho acontecimiento se refieren las primeras palabras que escribe en el diario mediante el cual reconstruye los acontecimientos y el proceso de duelo tras la muerte de su hijo:

7.292 DIAS DE PLENA FELICIDAD, 174.999 HORAS, 10.499.940 MINUTOS, 629.996.400 SEGUNDOS

1 de enero de 2011

08.00 am. Suena el frio teléfono y nos llaman desde el hospital, te han atropellado, disgustados pero confiados nos dirigimos al mismo, los nervios de urgencia, el informe de la cirujana y el traumatólogo, difícil pero con esperanzas, eres un luchador y confiamos en ti. Un par de largas horas de espera en quirófano y repitiendo y repitiéndome que cada minuto nos da más esperanza de que te quedes entre nosotros. Pero desgraciadamente llega la mala noticia te necesitan en otro sitio más que nosotros. Te necesitamos. Será verdad pero no sabes cómo duele, se suceden las llamadas informando a todo el mundo y los llantos de todo el mundo QUE GRANDE ERAS HIJO COMO TE

\footnotetext{
1 Los nombres y algunos de los detalles personales del informante, sus familiares y amigos que se presentan han sido modificados para preservar el anonimato y la confidencialidad.
} 
QUERIAN. Te secuestran durante todo un día en el anatómico forense y mientras volvemos a casa, Yo corro como un poseso a tu cuarto por que confió en que se han equivocado que tú no eres y estas durmiendo, pero no, no estás, y empieza el infierno, que comida sin querer, que tarde más larga y el teléfono sin parar, solo las pastillas no hacen conciliar el sueño, desde ese día son nuestras mejores aliadas para el descanso.

La vida marca el tempo del contexto, mientras, el tiempo subjetivo, espacializado, construye la dimensión real de la memoria de la pérdida, de manera que la experiencia vivida y rememorada es idéntica al espacio que acompaña permanentemente a los padres. En este sentido Benito construye mediado por su diario una historia de pérdida y de dolor a través de la cual sigue comunicándose con su hijo Josué, de modo que espacio y tiempo se comportan como "formas trascendentales (García et al, 2018:56). Así lo expresa en los siguientes fragmentos narrativos:

Querido amigo me permito extractar algunas de tus ideas de tu último mes, quitando lo cotidiano, la comida que es mucha (3 al día), el deporte y el Barça - Madrid. Gracias por permitirme hacerlo.

En tu diario hablas de tu identidad y del mundo de cómo ha cambiado tu visión de ambos a lo largo de este año, duro sin Josué y, de la necesidad de renegociar la forma en que seguir vinculado con el recuerdo de tu hijo de un modo en que puedas ver de nuevo el mundo al completo como lo veías y sentías antes. Una aspiración que espero y deseo que tengas más cerca de conseguir en el 2012 (mes de diciembre de 2011).

Benito incluye fragmentos que buscan un recuerdo perdurable, pleno de luz y sentido, que en ocasiones pugnan de un modo agridulce:

Hoy el sol ha salido para que le recordemos con su alegría siempre en nuestro recuerdo, Puri, Jesús, Maria y Raquel.

Quiero escribirte algo, pero no encuentro las palabras,

Quiero ver tu sonrisa, pero no encuentro tu boca, 
Quiero besar tu mejilla y no encuentro tu cara.

Y solo las encuentro en mi vida ya pasada.

Y otro día más sin verte, y van..., que decir sin causar daños a nadie, que callar sin que te dañes, me doy cuenta que a la gente le preocupa mis comentarios en Facebook, no quiero dar lastima a nadie, no es lo que pretendo, mi único objetivo es demostrar el inmenso cariño que le tengo a mis hijos, en ningún momento es un llanto por la ausencia de Josué, ya no me quedan lagrimas para derramar, solo espero una larga vida para recordar.

Verdeguea el barranco de los almendros blancos

Brotan los verdes tréboles, tras el estival descanso

Que se puede esperar de una vida frustrada por una decepción, por una losa pesada e inmanejable que tienes que cargar a tu espalda de por vida, que te lastra y para tu progresión diaria, esa es la definiciones de mi vida actual, sé que no puedo hacer nada más por mi hijo, sino recordarlo con amor y cariño, pero intento encontrar algo que me ayude en mi quehacer diario y no nada, solo puedo seguir viviendo de dos maneras, bien o mal, me gustaría elegir el bien, pero mi cabeza elige el mal y ante eso no encuentro ayuda posible.

Voy a correr y le pongo mis plantillas encima de las suyas en los tenis.

Mientras, tiempo y acción, vida y narrativas se dan la mano, se entrecruzan para dar sentido a lo vivido, incluso va más allá al relacionar lo que come o siente con el mundo de su hijo que configura un universo plagado de significados y sentimientos, en ocasiones contradictorios, pero que vinculan a Benito con su hijo Josué:

Compre una paleta de jamón ibérico como le gustaba a mi Josué. Van a ser las primeras navidades sin él y estoy nerviosísimo, tengo mucho respeto, miedo no tengo ya ni a la Muerte, cada día me vienen más recuerdos.

Vamos, vamos, vamos..., venga, venga, venga..., a seguir luchando para terminar de una vez este maldito año. 
Se me hace muy difícil el conjugar la alegría de los demás con mi melancolía.

Nos reunimos con otros padres que perdieron hijos y hemos estado hasta las 19,00 horas, vinieron Francisco y Mercedes, Julián y Suzi, Jonathan y señora, Petra y Carlos, Josefa, Felipe y Flora, y hemos pasado un rato muy agradable y nos hace mucho bien, pero me sigue faltando mi hijo.

En un camino que se torna una búsqueda al reencuentro con el que fue antes de la muerte del hijo. En un contexto que conoce y desea estar, plagado de recuerdos y objetos que añoran el pasado y que hablan de lo perdido, de la identidad. En la construcción o la búsqueda de una nueva identidad que se resiste a separarse de la que fue. Pues la de siempre se vive ahora algo como difusa, sin el hijo, e interroga permanentemente con la pregunta ¿cómo poder hacerlo sin él?

Que conste que no quiero ser un cabreado con el mundo, simplemente que no lo veo como me enseñaron que debía verlo, todo el mundo tiene derecho a ser feliz y no quiero cortar la felicidad de nadie, pero si me pase la vida dando, ¿es mucho pedir poder recibir algo sin tener que pedirlo? Quizás transmito una seguridad en mí mismo que impide a los demás el ayudarme más de lo que necesito y, siempre di sin ánimo de recibir. Lo único que quiero es encontrar otros yo que se den cuenta que detrás de esta fachada existe un corazón herido y con difícil, por no decir imposible cura. Voy a intentar leer este diario para hacer algo bonito para el aniversario de la partida de mi hijo, a ver si lo consigo. Nos estamos planteando que hacer en su memoria y cómo.

Hijo te eche mucho de menos, solo por no contagiar las lágrimas me las aguante todo el día.

Y los sentimientos se anclan y quedan ligados a los acontecimientos, a los lugares, a las fechas, a un calendario personal, cada vez marcado más por la ausencia.

Ya paso Nochebuena y Navidad, lógicamente los sentimientos están a flor de piel, han sido jodidos, pero seguimos vivos y vamos a seguir luchando, ya solo queda el fin de año y su aniversario, que será lo más fuerte pero seguro nos da fuerzas el 
mismo para seguir con la lucha, como hizo hasta ahora y así es más fácil, por lo menos eso creo, pero a veces del dicho al hecho va un trecho.

Hoy me levante positivo instare sacar solo lo positivo de mí, y dejare lo negativo, que bonito como intención pero mantenerlo a diario se me hace complicado, vamos a ver cómo sigue en los días sucesivos, pero hoy lo llevo a rajatabla, no puede ser de otra forma.

Todo un camino personal bajo la promesa de no sólo recordar al ser querido, sino de ser mejor persona, de mejorar como individuo y que querer ser esa nueva persona que ahora soy. Ser capaz de identificarme de nuevo con quien soy.

Hoy es el día de los inocentes, me gustaría que todo este año hubiera sido una broma, larga y macabra pero broma, de todas las maneras me planteo el dejar de llorar, el último día de llanto es el domingo día 1, en adelante solo fechas señaladas se acabó ser una plañidera, solo hay dos opciones en este proceso, una, que mi hijo este en otro mundo y esté sufriendo por vernos sufrir y dos que no exista nada y entonces porque nos preocúpanos, a donde van nuestros llantos y sufrimientos dirigido, a nada...

El deporte es el bálsamo que suaviza mis heridas, es ir a correr y volver completamente diferente a casa, pero a medida que pasa el día vuelve las cosquillas al estómago, quiero el día 1 hacer un punto y aparte, y dejar de dar lastima el próximo año, por lo menos no dejar aflorar los sentimiento o no tenerlos a flor de piel.

A lo largo de más de ciento cincuenta páginas Benito deambula por un mundo personal, mágico y cotidiano a la vez, de la mano del hilo conductor de la memoria de su hijo:

Me gustaría hacer un resumen de estas 159 páginas en las que escribo el devenir diario y un maremágnum de sentimientos que no se si fui capaz de transmitirlo como realmente los viví. Quería que fuera un homenaje a una gran persona, a un gran amigo, a un gran hijo que tuvo la mala suerte de salir a disfrutar la primera noche del año y no fue capaz de controlar la ingesta de alcohol y acabo 
debajo de un coche que por la mala suerte pasaba por allí con la sana intención de llevar a su dueña al trabajo. Desde ese día no solo no somos los mismos, tampoco conseguimos recuperar la normalidad anterior. Si no estamos todos en casa, ya vienen los malos pensamientos. Ya cualquier chico joven con melenita nos recuerda a nuestro Josué, ya no oigo el Carlos llamándolo “loco". Nos falta alegría. No sé si esté próximo año voy a seguir escribiendo, lo mismo lo hago pero sin la esclavitud diaria.

Sin faltar a esa promesa eterna y permanente de "te recordaré siempre" las formas y modos de seguir conectado con el hijo están presentes en los actos y la vida cotidiana de Benito.

Ya ha pasado un año. ¡Cómo pasa el tiempo...! Aunque intente con una escalera llegar al cielo y poder abrazarte no conseguí ese deseo, y la línea de teléfono directa al paraíso estaba apagada o fuera de cobertura y no pudimos hablar, pero te mando un beso fuerte y nuestra promesa de que no te olvidaremos. SOLO SE MUERE CUANDO NO EXITE EL RECUERDO.

Mientras, la vida por venir se construye sin la presencia física del hijo, su representación social y los acercamientos a él se dan mediados por espacios y lugares, mediado por conversaciones, por el diario personal y el Facebook. A los 100 días del fallecimiento Josué, Benito escribe en su Facebook un precioso fragmento mezcla de dolor, nostalgia y anhelo de rencuentro:

\section{EN MEMORIA}

de Benito García Estrada. Domingo, 10 de abril de 2011 a las 10:51.

10 de abril de 2011, 100 días ya hoy, 100 espinas clavadas en mi corazón, desde ese día, en eso se convierten mis días, en espinas. Cada amanecer es una nueva espina que se clava en mi corazón al ver tu cuarto vacío, y solo el amor de tu madre y hermano me dan 100 razones para seguir viviendo, porque 100 veces habría dado mi vida a cambio de la tuya y así tendrá que ser hasta que llegue el momento, espero que lejano, en que nos encontremos ese lugar donde vayamos.

Acontecimiento de marcado sentido simbólico como una misa dedicada a su hijo le llevan a mencionarlo y publicarlo en el Facebook: 
HIJO EL NO VERTE, AUMENTA PROPORCIONALMENTE EL AMOR QUE TE TENGO y la misa me dejó una gran paz interior, como que está haciendo algo más importante que contribuir a nuestra felicidad, te estamos compartiendo con más gente" (29 de julio de 2011).

Se fueron demasiado pronto... O se fueron simplemente. Cuando miramos al cielo nos gusta pensar que miran por nosotros. A menudo nos acordamos de ellos... Por la mañana... Por la noche mirando las estrellas... Una fecha... Una canción... Un lugar... Un olor... En memoria de los que nos dejaron, copia esto en tu muro si extrañas a alguien que hoy te mira desde el cielo" (20 de agosto de 2011).

La muerte no nos roba a los seres amados. Al contrario, nos los guarda y nos los inmortaliza en el recuerdo. La vida sí que los roba muchas veces definitivamente" (1 de enero de 2012, primer aniversario del fallecimiento de Josué).

Ya un año, como pasa el tiempo, aunque intente con una escalera llegar al cielo y poder abrazarte, no conseguí ese deseo, y la línea de teléfono directa al paraíso estaba apagada o fuera de cobertura y no pudimos hablar, pero te mando un beso fuerte y nuestra promesa de que no te olvidaremos. SOLO SE MUERE CUANDO NO EXISTE EL RECUERDO" (1 de enero de 2012).

1826 días st snc Josué (1 de enero de 2016).

Para siempre en nuestros corazones, Josué" (1 de enero de 2017)

El 21 de junio de 2018, fecha del 28 cumpleaños de Josué, escribe Benito en el Facebook:

"Feliz cumpleaños, Te queremos mucho", acompañado del video del grupo Maná interpretando en directo la canción "Si no te hubieras ido".

El futuro simbólico de los padres en duelo y la continuidad de vínculos entre ellos y sus hijos se corresponde a su pasado simbólico con el que guarda una estricta analogía, y al futuro que podemos denominar "profético", pues en ningún lugar encuentra mejor expresión que en la vida de los grandes profetas religiosos, quienes 
nunca se contentaron con comunicar simplemente futuros sucesos o con advertir sobre daños posibles y que, al igual que los padres, no hablaban como augures ni aceptaban la evidencia de los omina y los presagios. Un propósito opuesto al de los adivinos. El futuro del que habla Benito, al igual que otros padres que perdieron hijos se comporta más como un hecho empírico que como una tarea ética y religiosa, una predicción que desean se transforme en profecía, profecía que no significa predicción, sino promesa. Su futuro ideal busca la negación del mundo empírico, "el fin de los tiempos", y contiene, al mismo tiempo, la esperanza y la seguridad de un nuevo ciclo y de una nueva tierra. De nuevo los padres, tal como los profetas se atreven más allá de los límites de la existencia finita por seguir unidos a su ser querido (García, 2012: 95-96).

La continuidad de lazos con el hijo da consuelo y ayuda a dar una visión duradera del mundo, en el que la construcción y el mantenimiento de estos lazos "interiores" y "exteriores" sirven de cura al individuo y la comunidad y les permiten no sólo aceptar la muerte y asumirla, sino más aún, ordenarla, integrándola a su sistema cultural, lo cual no deja de ser la mejor manera de dominarla, imitarla ritualmente en la iniciación y, trascenderla gracias a un juego apropiado y complejo de símbolos personales (Field y otros, 2005; Klass, 2006; García et al., 2016; García, 2018: 139).

\section{Bibliografía}

Field, N. P., Gao, B. I., Paderna, L. Continuing bonds in bereavement: An attachment theory based perspective. Death studies, 29, 2005, pp. 277-299.

García, A. M. La pérdida de un hijo y la búsqueda de significado: Reescribiendo historias de pérdida y de dolor. En W. Astudillo, A. Ispizua, A. Orbegozo (Eds.), Acompañamiento en el duelo y Medicina Paliativa, pp. 133-156. San Sebastián, Sociedad Vasca de Cuidados Paliativos, 2007. 
García, A. M. Duelo y significados. En W. Astudillo, A. Ispizua, A. Orbegozo (Eds.), Acompañamiento en el duelo y Medicina Paliativa, pp. 117-134. San Sebastián, Sociedad Vasca de Cuidados Paliativos, 2007.

García, A. M. Continuidad de lazos entre madres y padres y sus hijos fallecidos. Experiencia con padres participantes en un grupo de duelo. ENE, 3, 2008, pp. 34-44. García, A. M. El significado de perder un hijo: la construcción discursiva del duelo de padres y madres. Tenerife, Universidad de la Laguna, 2010.

García, A. M. La pérdida y el duelo. Una experiencia compartida. España, Editor Bubok Publishing S. L., 2012.

García-Hernández, AM, Rodríguez M, Brito PR. Object repertoires that evoke memories for mothers and fathers who have lost children. Tenerife. Spain. Majorensis, 12, 2016, pp. 12-21.

García-Hernández, A.M. Narrativas y continuidad de vínculos en padres que perdieron hijos. Majorensis, 13, 2017, pp. 118-125.

García, A.M.; Rodríguez, M.; Brito, PR. Duelo de padres tras la muerte de un hijo. En Medicina Paliativa en niños y adolescentes. W. Astudillo, I. Astigarraga, A Salinas, C. Mendinueta, A. Navajas, C. D’Souza y S. Jassal. (Editores), Paliativos sin fronteras, San Sebastián. España. 2015. pp. 631-639.

García, A.M. El duelo. En Enfermería Comunitaria II, 3aㅡ Edición de la Colección Enfermería Siglo 21. Editorial DAE, Grupo Paradigma, 2016, pp. 901-918.

García, A.M. La experiencia de perder un hijo. Amazon.es, 2018.

García, A.M. Duelo y causalidad. Amazon.es, 2019.

García, A.M.; Rodríguez, M.; Brito, P.R.; Peyrolón, J. Continuidad de vínculos y duelo: Nuevas perspectivas. Health, Aging \& End of life, 3, pp. 49-65.

Klass, D. Continuing conversation about continuing bonds. Death Studies, 30(9), 2006, pp. 843-858. 
Torres, C.; García, AM. From Violation to Voice, from pain to protest. Healing and transforming unjust loss through the use of rituals and memorial. En Handbook of Social justice in loss and grief. Exploring Diversity, Equity, and Inclusion. Darcy L. Harris y Tashel C. Bordere (Editoras). Series in Death, Dying, and Bereavement. Routledge Taylor \& Francis Group. New York and London, 2016. pp. 201-212. 